\title{
MULTIPARTITIONS, GENERALIZED DURFEE SQUARES AND AFFINE LIE ALGEBRA CHARACTERS
}

\author{
PETER BOUWKNEGT
}

\author{
Dedicated to Rodney J. Baxter on his 60th birthday
}

(Received 26 April 2000)

Communicated by I. Aitchison

\begin{abstract}
We give some higher dimensional analogues of the Durfee square formula and point out their relation to dissections of multipartitions. We apply the results to write certain affine Lie algebra characters in terms of Universal Chiral Partition Functions.
\end{abstract}

2000 Mathematics subject classification: primary 05A17, 05A19, 17B67, 81T40.

\section{Introduction and background}

In this paper we will consider certain generalizations of an identity, due to Euler, known as the Durfee square identity (see [3] for an excellent introduction and historical account)

$$
\frac{1}{(q)_{\infty}}=\sum_{m \geq 0} \frac{q^{m^{2}}}{(q)_{m}(q)_{m}}
$$

where

$$
(z ; q)_{M}=\prod_{k=1}^{M}\left(1-z q^{k-1}\right), \quad(q)_{M} \equiv(q ; q)_{M} .
$$

There are various ways to prove this identity. For instance, it follows as a limiting case of the $q$-analogue of Gauss' formula for the basic hypergeometric series ${ }_{2} \phi_{1}$ (see,

The author was supported by a QEII research fellowship from the Australian Research Council.

(c) 2002 Australian Mathematical Society 1446-7887/2000\$A2.00+0.00 


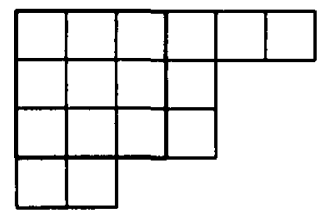

FIGURE 1.1. The partition $\lambda=(6,4,4,2)$ and its $3 \times 3$ Durfee square

for example, [2]). The most lucid proof, however, employs the connection of (1.1) to partitions [10] (see also $[8,2])$. Henceforth we identify partitions $\lambda=\left(\lambda_{1}, \lambda_{2}, \ldots\right)$, $\lambda_{1} \geq \lambda_{2} \geq \ldots \geq 1$, and their graphical presentation in terms of Young diagrams [2] (see, for example, Figure 1.1 for the partition $\lambda=(6,4,4,2)$ ).

Now, recall that

$$
(z q)_{M}^{-1}=\sum_{m, n \geq 0} p_{M}(m, n) z^{m} q^{n}
$$

where $p_{M}(m, n)$ denotes the number of partitions of $n$ into $m$ parts in which no part exceeds $M$. In terms of Young diagrams, $p_{M}(m, n)$ is the number of diagrams with $n$ boxes such that there are $m$ rows and no more than $M$ columns.

Thus, the left-hand side of (1.1) is clearly the generating function for all partitions, while each summand on the right-hand side correspond to all partitions which fit at most an $m \times m$ 'Durfee square' in the upper left-hand corner of the Young diagram. (The $3 \times 3$ Durfee square for the partition $\lambda=(6,4,4,2)$ is indicated in Figure 1.1.) Summing over all $m$ clearly generates the total set of partitions as well. This proves (1.1). In fact, by keeping track of the number of columns and rows in the above argument we have the following generalization of (1.1) due to Cauchy

$$
\frac{1}{(z q)_{M}}=\sum_{m \geq 0} \frac{q^{m^{2}} z^{m}}{(z q)_{m}}\left[\begin{array}{c}
M \\
m
\end{array}\right]
$$

where

$$
\left[\begin{array}{l}
m \\
n
\end{array}\right]=\frac{(q)_{m}}{(q)_{n}(q)_{m-n}}
$$

for $0 \leq n \leq m$ (and zero otherwise), denotes the $q$-binomial (Gaussian polynomial).

Instead of dissecting partitions according to their maximal Durfee square, Andrews considered dissections by (maximal) rectangles whose base to height ratio is $r: s$ and obtained the following generalization of (1.4) [1]

$$
\frac{1}{(z q)_{M}}=\sum_{i, j} \sum_{m \geq 0} \frac{q^{(r m+i)(s m+j)} z^{r m+i}}{(z q)_{s m+j-1+\delta_{i, 0}+\delta_{i, r}}}\left[\begin{array}{c}
M+r m+i \delta_{j, s}-s m-j \\
r m+i \delta_{j, s}
\end{array}\right],
$$


where the sum over $(i, j)$ is over all pairs

$$
(i, j) \in\{(i, j)=(0,0) \text { or } 1 \leq i \leq r, 1 \leq j \leq s,(i, j) \neq(r, s)\} .
$$

In fact, the identity (1.6) is valid even if $r$ and $s$ are not relatively prime, as is obvious from Andrews' proof. For $(r, s)=(1,1),(1.6)$ reduces to (1.4), while for $(r, s)=(2,1)$ it gives an identity which appears explicitly in Ramanujan's lost notebook (see [3]). The proof of (1.6) was also sketched by Berkovich and McCoy in [6], and the $M \rightarrow \infty$ limit of (1.6) was discussed by van Elburg and Schoutens in [7].

In this paper we will consider further generalizations of (1.6) by considering simultaneous dissections of multipartitions. The resulting formulas are useful in deriving expressions for the chiral characters of $2 \mathrm{D}$ conformal field theories (in particular the characters of modules of affine Lie algebras) in terms of so-called universal chiral partition functions (UCPF's).

\section{Durfee systems}

We will be concerned with identities of the form

$$
\begin{aligned}
\frac{1}{\prod_{i}\left(z_{i} q\right)_{M_{i}}}= & \sum_{k} \sum_{\substack{m \in Z_{+}^{n} \\
n-K \cdot m=Q^{(k)}}}^{\prime}\left(\prod_{i} z_{i}^{m_{i}+a_{i}^{(k)}}\right) q^{\left(m+a^{(k)}\right) \cdot\left(n+b^{(k)}\right)} \frac{1}{\prod_{i}\left(z_{i} q\right)_{n_{i}}} \\
& \times \prod_{i}\left[\begin{array}{c}
M_{i}+m_{i}-\left(n_{i}+b_{i}^{(k)}\right) \\
m_{i}
\end{array}\right],
\end{aligned}
$$

where $K \in G L(n, \mathbb{Q})$ is a symmetric matrix and the sum over $k$ is over a (finite) set of sectors. In each sector $k$, the sum over $m$ is over those $m \in\left(\mathbb{Z}_{+}\right)^{n}$ (here $\mathbb{Z}_{+}$denotes the set of non-negative integers) such that $K \cdot \boldsymbol{m}+Q^{(k)} \in\left(\mathbb{Z}_{+}\right)^{n}$, while $n=K \cdot \boldsymbol{m}+\boldsymbol{Q}^{(k)}$.

DEFINITION 2.1. A Durfee system for $\boldsymbol{K} \in G L(n, \mathbb{Q})$, of length $L$, is a collection of $n$-dimensional vectors, $\left(Q^{(k)}, a^{(k)}, b^{(k)}\right), k=0, \ldots, L-1$, such that $(2.1)$ is satisfied for all $M_{i} \in \mathbb{Z}_{+}$and $z_{i}(i=1, \ldots, n)$.

Andrews' $(r, s)$-generalization of the classical Durfee formula, discussed in Section 1 , can now be formulated as

THEOREM 2.2. Let $r, s \in \mathbb{N}$. A Durfee system of length $L=r s$, for the $1 \times 1$ matrix $K=s / r$, is given by

$$
\begin{aligned}
& Q^{(i, j)}=j-1+\delta_{i, 0}+\delta_{i, r}-\frac{s}{r} i \delta_{j, s}, \\
& a^{(i, j)}=i\left(1-\delta_{j, s}\right), \quad b^{(i, j)}=1-\delta_{i, 0}-\delta_{i, r},
\end{aligned}
$$

where $k=(i, j)$ runs over the rs sectors as in (1.7). 
In the remainder of this paper we restrict ourselves to non-negative integer-valued, symmetric matrices $K$, that is, $K \in G L\left(n, \mathbb{Z}_{+}\right)$, and Durfee systems $\left(Q^{(k)}, a^{(k)}, b^{(k)}\right)$ of $n$-vectors with entries in $\mathbb{Z}_{+}$. In this case the sum in (2.1) is over all $m_{i} \geq 0$ and $n_{i} \in \mathbb{Z}_{+}$is determined by $n=K \cdot \boldsymbol{m}+\boldsymbol{Q}^{(k)}$.

Before giving examples, let us first explore some consequences of (2.1). By replacing $z_{i} \rightarrow z_{i} q^{p_{1}}$ in (2.1), for some $p \in \mathbb{Z}^{n}$, using the expansion (Note that (2.3) itself can be interpreted as a length- 1 Durfee system for the trivial matrix $K=0$ with $(Q, a, b)=(0,0,1)$.)

$$
\frac{1}{(z q)_{M}}=\sum_{m \geq 0}(z q)^{m}\left[\begin{array}{c}
M+m-1 \\
m
\end{array}\right]
$$

and shifting the summation variables, we find

$$
\begin{aligned}
\prod_{i}\left[\begin{array}{c}
M_{i}+N_{i} \\
M_{i}
\end{array}\right]= & \sum_{k} \sum_{\substack{m \in \mathbb{Z}_{+}^{n} \\
n-K \cdot m=Q^{(k)}+p}} q^{\left(m+a^{(k)}\right) \cdot\left(n+b^{(k)}\right)} \prod_{i}\left[\begin{array}{c}
M_{i}+m_{i}-\left(n_{i}+b_{i}^{(k)}\right) \\
m_{i}
\end{array}\right] \\
& \times\left[\begin{array}{c}
N_{i}+n_{i}-\left(m_{i}+a_{i}^{(k)}\right) \\
n_{i}
\end{array}\right],
\end{aligned}
$$

for arbitrary $p \in \mathbb{Z}^{n}$. Note that in this formula the summation variables $(m, n)$ appear on a more symmetrical footing.

By taking the limit $M_{i} \rightarrow \infty$ in (2.1) we find

$$
\frac{1}{\prod_{i}\left(z_{i} q\right)_{\infty}}=\sum_{k} \sum_{\substack{\boldsymbol{m} \in Z_{+}^{n} \\ n-\boldsymbol{K} \cdot \boldsymbol{m}=\boldsymbol{Q}^{(k)}}}\left(\prod_{i} z_{i}^{m_{i}+a_{i}^{(k)}}\right) \frac{q^{\left(m+a^{(k)} \cdot\left(n+b^{(k)}\right)\right.}}{\prod_{i}(q)_{m_{i}}\left(z_{i} q\right)_{n_{i}}},
$$

while by specializing (2.5) to $z_{i}=q^{p_{i}}$, we find a generalization of the classical Durfee formula (1.1)

$$
\frac{1}{(q)_{\infty}^{n}}=\sum_{k} \sum_{n-K \cdot m=Q^{(k)}+p} \frac{q^{\left(m+a^{(k)}\right) \cdot\left(n+b^{(k)}\right)}}{\prod(q)_{m_{i}}(q)_{n_{l}}}
$$

for any constant vector $p \in \mathbb{Z}^{n}$. Of course, this equation can also be obtained from (2.4) by letting all $M_{i} \rightarrow \infty$. Other interesting formulas are obtained by taking different specializations of (2.4).

The search for identities of the type (2.1) in dimension $n$ is greatly facilitated by using results in lower dimensions. Indeed, by putting $z_{i}=0$ for some $i=i_{0}$ in (2.1), the right-hand side only receives contributions from the sectors $k$ for which $a_{i_{0}}^{(k)}=0$. For those sectors only the term $m_{i_{0}}=0$ contributes in the summation, and 


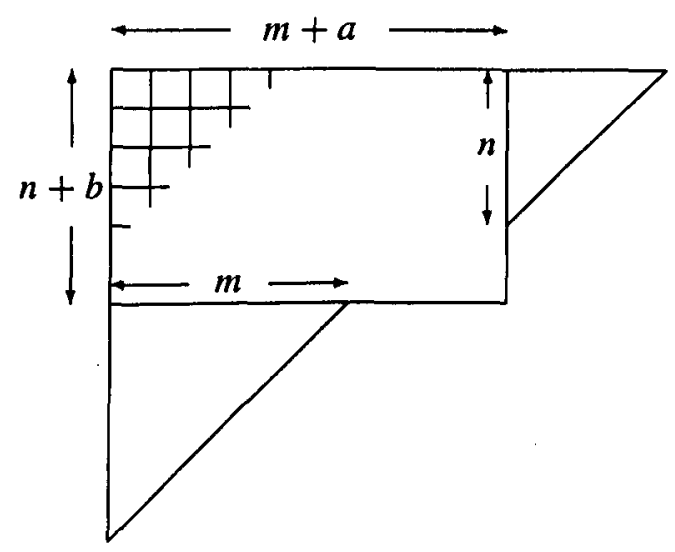

FIGURE 2.1. Set of partitions with generating function (2.7)

(2.1) reduces to a similar identity in dimension $n-1$. Summarizing, if we know identities for a $(n-1) \times(n-1)$ sub-block of $K$, then we learn about the components $\left(Q_{i}^{(k)}, a_{i}^{(k)}, b_{i}^{(k)}\right), i \neq i_{0}$, for all sectors $k$ for which $a_{i_{0}}^{(k)}=0$.

We now discuss the correspondence of Durfee systems with multipartitions. Suppose we have a Durfee system $\left(\boldsymbol{Q}^{(k)}, \boldsymbol{a}^{(k)}, \boldsymbol{b}^{(k)}\right)$ for $\boldsymbol{K} \in G L\left(n, \mathbb{Z}_{+}\right)$. Consider (2.6) for $p=0$. The left-hand side is the generating series for all multipartitions $\left(\lambda^{(1)}, \lambda^{(2)}, \ldots, \lambda^{(n)}\right)$. Each term in the summand on the right-hand side of $(2.6)$ is a product (over $i$ ) of terms of the form

$$
\frac{q^{(m+a)(n+b)}}{(q)_{m}(q)_{n}}
$$

By associating to (2.7) a set of partitions of the form indicated in Figure 2.1, each term in the summand on the right-hand side of $(2.6)$ is in $1-1$ correspondence with a set of multipartitions.

One possible strategy for proving the existence of a Durfee system is therefore to show that the set of $n$-dimensional multipartitions corresponding to the right-hand side of (2.6) is non-overlapping and exhaustive. By keeping track of the number of rows and columns in each partition $\lambda^{(i)}$, the generalization (2.1) then easily follows.

After discussing some examples of Durfee systems in the following sections we will explore some further consequences in the context of affine Lie algebra characters.

\section{Examples}

In this section we will consider some examples of Durfee systems. 

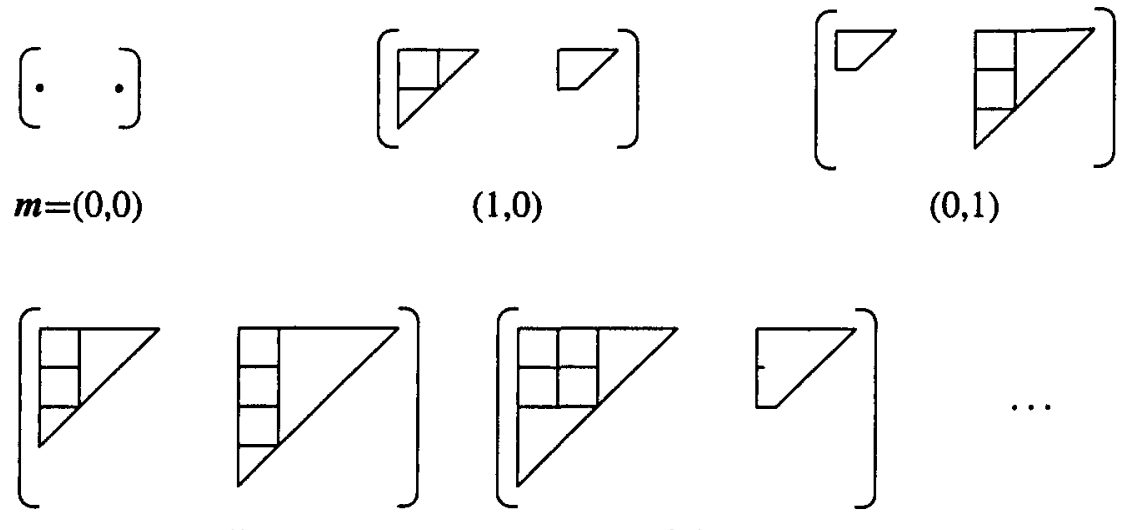

$(0,1)$

$(1,1)$

$(2,0)$

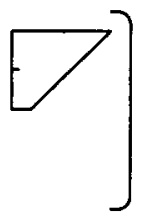

FIGURE 3.1. The $k=0$ sector

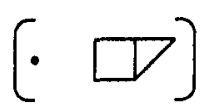

FIGURE 3.2. Some missing bipartitions

THEOREM 3.1. Consider the matrix $K \in G L\left(2, \mathbb{Z}_{+}\right)$given by

$$
\boldsymbol{K}=\left(\begin{array}{ll}
1 & 1 \\
1 & 2
\end{array}\right)
$$

We have a Durfee system $\left(\boldsymbol{Q}^{(k)}, \boldsymbol{a}^{(k)}, \boldsymbol{b}^{(k)}\right)$ for $\boldsymbol{K}$ given by

$$
\begin{array}{lll}
\boldsymbol{Q}^{(0)}=\left(\begin{array}{l}
0 \\
0
\end{array}\right), & \boldsymbol{a}^{(0)}=\left(\begin{array}{l}
0 \\
0
\end{array}\right), & \boldsymbol{b}^{(0)}=\left(\begin{array}{l}
0 \\
0
\end{array}\right), \\
\boldsymbol{Q}^{(1)}=\left(\begin{array}{l}
0 \\
1
\end{array}\right), & \boldsymbol{a}^{(1)}=\left(\begin{array}{l}
0 \\
1
\end{array}\right), & \boldsymbol{b}^{(1)}=\left(\begin{array}{l}
1 \\
0
\end{array}\right) .
\end{array}
$$

Let us illustrate, in some detail, how one might arrive at this result. The $k=0$ term in (2.6) (for $p=0$ ) is explicitly given by

$$
\sum_{\substack{n_{1}-\left(m_{1}+m_{2}\right)=0 \\ n_{2}-\left(m_{1}+2 m_{2}\right)=0}} \frac{q^{n_{1} m_{1}+n_{2} m_{2}}}{(q)_{n_{1}}(q)_{n_{2}}(q)_{m_{1}}(q)_{m_{2}}} .
$$

The set of bipartitions $\left(\lambda^{(1)}, \lambda^{(2)}\right)$ associated to (3.2), according to the prescription of Section 2, is depicted in Figure 3.1 for low values of $\boldsymbol{m}=\left(m_{1}, m_{2}\right)$. 
[. $\square]$ $(0,0)$

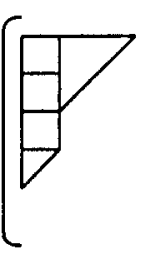

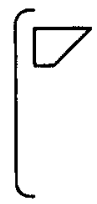

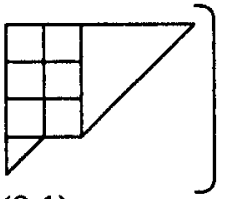

$(0,1)$

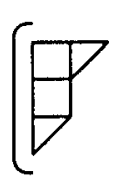

$(1,0)$

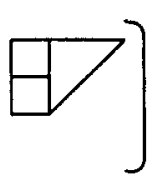

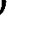

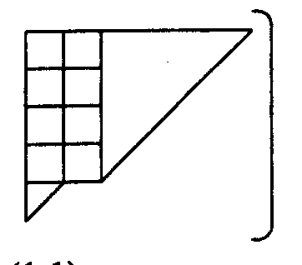

$(1,1)$

FIGURE 3.3. The $k=1$ sector

[.

$(0,0)$

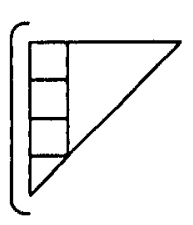

$(1,1)$

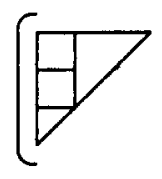

$(1,0)$
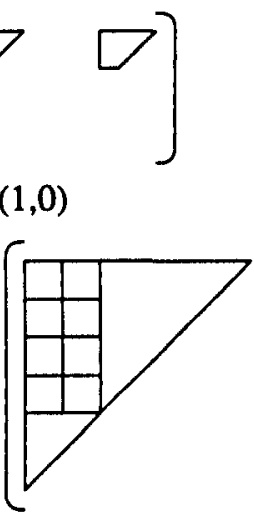

$(2,0)$
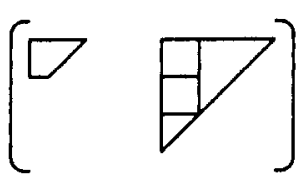

$(0,1)$

FIGURE 3.4. The $k=0$ sector

[. $\square]$

$(0,0)$

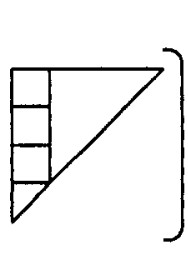

)

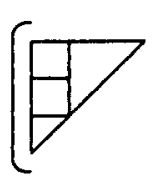

$(1,0)$

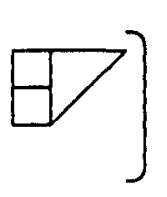

)

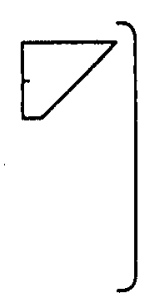

$\int$ 


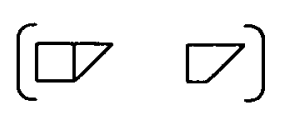

$(0,0)$

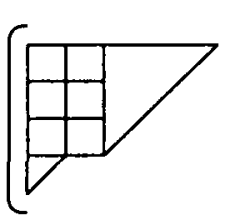

$(1,0)$
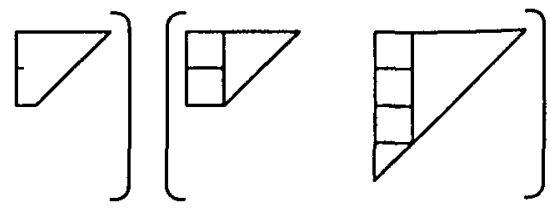

$(0,1)$

FIGURE 3.6. The $k=2$ sector

Clearly these do not exhaust the set of all bipartitions. For instance, if $\lambda^{(1)}=\emptyset$ (indicated by $a \bullet$ in Figure 3.1) and $\lambda^{(2)} \neq \emptyset$, then $\lambda^{(2)}$ necessarily has two or more rows. Thus, the set of bipartitions depicted in Figure 3.2 is missing in (3.2).

If this set of bipartitions is to be included as the $m=(0,0)$ term of another sector, say $k=1$, then this immediately fixes all components of $\left(Q^{(1)}, a^{(1)}, b^{(1)}\right)$ with the exception of $b_{1}^{(1)}$. (Note that this component is also unconstrained by consideration of the two $1 \times 1$ sub-blocks of $K$, as discussed in Section 2.) Consideration of the $m=(1,0)$ term in the $k=1$ sector, however, uniquely fixes $b_{1}^{(1)}$ as well and we arrive at the conclusion that (3.2) needs to be supplemented by

$$
\sum_{\substack{n_{1}-\left(m_{1}+m_{2}\right)=0 \\ n_{2}-\left(m_{1}+2 m_{2}\right)=1}} \frac{q^{\left(n_{1}+1\right) m_{1}+n_{2}\left(m_{2}+1\right)}}{(q)_{n_{1}}(q)_{n_{2}}(q)_{m_{1}}(q)_{m_{2}}} .
$$

The set of bipartitions in the $k=1$ sector, arising from (3.3) for low values of $m$, is depicted in Fig. 3.3.

Together, the sets of bipartitions of Figures 3.1 and 3.3 are seen to be nonoverlapping and to exhaust the set of all bipartitions, at least to low order, so it seems that no other sectors are required. The proof that this works to all orders requires a bit more work and will be omitted.

A slightly more complicated Durfee system is given in

THEOREM 3.2. Let

$$
K=\left(\begin{array}{ll}
2 & 1 \\
1 & 2
\end{array}\right)
$$

The following constitutes a Durfee system for $K$

$$
\begin{array}{lll}
Q^{(0)}=\left(\begin{array}{l}
0 \\
0
\end{array}\right), & a^{(0)}=\left(\begin{array}{l}
0 \\
0
\end{array}\right), & b^{(0)}=\left(\begin{array}{l}
0 \\
0
\end{array}\right), \\
Q^{(1)}=\left(\begin{array}{l}
0 \\
1
\end{array}\right), & a^{(1)}=\left(\begin{array}{l}
0 \\
1
\end{array}\right), & b^{(1)}=\left(\begin{array}{l}
0 \\
0
\end{array}\right), \\
Q^{(2)}=\left(\begin{array}{l}
1 \\
1
\end{array}\right), & a^{(2)}=\left(\begin{array}{l}
1 \\
0
\end{array}\right), & b^{(2)}=\left(\begin{array}{l}
0 \\
0
\end{array}\right) .
\end{array}
$$


The reasoning parallels that of Theorem 3.1. The first few sets of contributing bipartitions, for the sectors $k=0,1,2$, are depicted in Figures 3.4-3.6, respectively.

Theorem 3.2 has the following higher dimensional generalization

THEOREM 3.3. Let $K \in G L\left(n, \mathbb{Z}_{+}\right)$be defined by

$$
\boldsymbol{K}=\left(\begin{array}{cccc}
2 & 1 & \cdots & 1 \\
1 & 2 & \cdots & 1 \\
\vdots & \vdots & \ddots & \vdots \\
1 & 1 & \cdots & 2
\end{array}\right)
$$

We have a Durfee system of length $L=n+1$, given by the $n$-vectors

$$
\begin{aligned}
Q^{(k)} & =(\underbrace{0,0, \ldots, 0}_{n-k}, \underbrace{1, \ldots, 1}_{k}), \\
\boldsymbol{a}^{(k)} & =(\underbrace{0, \ldots, 0}_{n-k}, 1 \underbrace{0, \ldots, 0}_{k-1}), \quad b^{(k)}=(\underbrace{0,0, \ldots, 0}_{n}),
\end{aligned}
$$

for $k=0, \ldots, n$.

REMARK. Note that the length of the Durfee system in Theorem 3.3 is given by $L=n+1=\operatorname{det} \boldsymbol{K}$. We believe this is a general feature of Durfee systems for which $\boldsymbol{b}^{(k)}=0$ for all $k=0, \ldots, L-1$ (see also the discussion in Section 6).

\section{Shift operation}

It turns out that, once a Durfee system for some $K \in G L\left(n, \mathbb{Z}_{+}\right)$has been established, it is rather straightforward to obtain a Durfee system for a class of deformations of $K$. These deformations are given in terms of a 'charge vector' $t=\left(t_{1}, \ldots, t_{n}\right)$, $t_{i} \in \mathbb{Z}_{+}$, and a positive integer $M \in \mathbb{Z}_{+}$as (These deformations were motivated by the 'shift operation' on $K$-matrices describing fractional quantum Hall systems (see [5] and references therein).)

$$
K_{M, t}=K+M t t^{T} .
$$

For instance, consider the deformation $\boldsymbol{K}_{M, t}$ of the two-dimensional identity matrix

$$
K_{M, t}=\left(\begin{array}{cc}
t_{1}^{2} M+1 & t_{1} t_{2} M \\
t_{1} t_{2} M & t_{2}^{2} M+1
\end{array}\right)
$$

where we can assume that $t_{1} \leq t_{2}$. Note that the matrix $K$ of (3.4) is of this form with $M=1, t=(1,1)$. 
THEOREM 4.1. The matrix $K_{M, t}$ of (4.2) admits a length $L=\left(t_{1}^{2}+t_{2}^{2}\right) M+1$ Durfee system. There are $t_{2}^{2} M$ sectors given by

$$
\begin{aligned}
Q= & \underbrace{}_{\left(\begin{array}{c}
\left(_{2}^{2}-t_{1}^{2}\right) M \\
t_{2}^{2} M
\end{array}\right),\left(\begin{array}{c}
t_{1}^{2} M+1 \\
t_{2}^{2} M-1
\end{array}\right), \ldots,\left(\begin{array}{c}
t_{1}^{2} M+1 \\
t_{1}^{2} M+2
\end{array}\right)} \\
& \underbrace{\left(\begin{array}{c}
t_{1}^{2} M \\
t_{1}^{2} M+1
\end{array}\right),\left(\begin{array}{c}
t_{1}^{2} M-1 \\
t_{1}^{2} M
\end{array}\right), \ldots,\left(\begin{array}{l}
0 \\
1
\end{array}\right)}_{t_{1}^{2} M},
\end{aligned}
$$

with $a=\left(\begin{array}{l}0 \\ 0\end{array}\right), b=\left(\begin{array}{l}0 \\ 0\end{array}\right), t_{1}^{2} M$ sectors given by

$$
Q=\left(\begin{array}{c}
t_{1}^{2} M \\
t_{1}^{2} M
\end{array}\right),\left(\begin{array}{c}
t_{1}^{2} M-1 \\
t_{1}^{2} M-1
\end{array}\right), \ldots,\left(\begin{array}{l}
1 \\
1
\end{array}\right)
$$

with $\boldsymbol{a}=\left(\begin{array}{l}1 \\ 0\end{array}\right), \boldsymbol{b}=\left(\begin{array}{l}0 \\ 0\end{array}\right)$, and and the 'vacuum sector' $\boldsymbol{Q}=\left(\begin{array}{l}0 \\ 0\end{array}\right), \boldsymbol{a}=\left(\begin{array}{l}0 \\ 0\end{array}\right), \boldsymbol{b}=\left(\begin{array}{l}0 \\ 0\end{array}\right)$.

For deformations (4.1), with $K=\mathbb{1}$, we have

$$
\operatorname{det} K_{M, t}=\left(t^{T} \cdot t\right) M+1,
$$

which can be written as

$$
\operatorname{det} K_{M, 1}=\operatorname{Tr}\left(K_{M, t}-1\right)+1 .
$$

In fact, if $n=2$, the matrix $K_{M, t}=1+M t t^{T}$ is the most general symmetric, nonnegative integer-valued matrix satisfying (4.6). Note that the length of the Durfee system in Theorem 4.1 is again given by $\operatorname{det} K_{M, 1}$.

\section{The UCPF and character identities}

Consider the 'Universal Chiral Partition Function' (UCPF) (see [6] and references therein)

$$
Z(\boldsymbol{K} ; \boldsymbol{Q}, \boldsymbol{u} \mid z ; q)=\sum_{\boldsymbol{m} \in \mathbb{Z}_{+}^{n}}\left(\prod_{i} z_{i}^{m_{i}}\right) q^{\frac{1}{2} m \cdot \boldsymbol{K} \cdot m+\boldsymbol{Q} \cdot m} \prod_{i}\left[\begin{array}{c}
((1-K) \cdot \boldsymbol{m}+\boldsymbol{u})_{i} \\
m_{i}
\end{array}\right],
$$

where $K \in G L\left(n, \mathbb{Z}_{+}\right), Q_{i} \in \mathbb{Z}_{+}$and $u_{i} \in \mathbb{Z}_{+} \cup\{\infty\}, i=1, \ldots, n$. (The considerations in this section can easily be generalized to triples $(K ; Q, u)$ with entries in $\mathbb{Q}$, provided appropriate restrictions on the summation variables $m_{i}$ in (5.1) are made.)

The following theorem is derived by elementary algebra 
THEOREM 5.1. Assume that $\left(Q^{(k)}, a^{(k)}, b^{(k)}\right)$ forms a Durfee system for a symmetric $K \in G L\left(n, \mathbb{Z}_{+}\right)$. Define

$$
Q^{\prime(k)}=-K^{-1} \cdot Q^{(k)}, \quad z_{i}^{\prime}=\prod_{j} z_{j}^{-K_{i j}}
$$

Then we have the following identity

$$
\begin{gathered}
\sum_{k}\left(\prod_{i} z_{i}^{-Q_{i}^{(k)}}\right) q^{\frac{1}{2} Q^{(k)} \cdot \boldsymbol{K}^{-1} \cdot Q^{(k)}+a^{(k)} \cdot b^{(k)}} Z\left(K ; Q^{(k)}+b^{(k)}, M-\left(Q^{(k)}+b^{(k)}\right) \mid z^{\prime} ; q\right) \\
\times Z\left(K^{-1} ; Q^{\prime(k)}+a^{(k)}, N-\left(Q^{\prime(k)}+a^{(k)}\right) \mid z ; q\right) \\
\quad=\sum_{p \in Z^{n}}\left(\prod_{i} z_{i}^{p_{i}}\right) q^{\frac{1}{2} \boldsymbol{p} \cdot K^{-1} \cdot p} \prod_{i}\left[\begin{array}{c}
M_{i}+N_{i}+\left(\left(\mathbb{1}-K^{-1}\right) \cdot \boldsymbol{p}\right)_{i} \\
M_{i}+p_{i}
\end{array}\right]
\end{gathered}
$$

for all $\boldsymbol{M}, N \in \mathbb{Z}_{+}^{n}$.

REMARK. Note that the polynomials $P_{M}^{(k)}(z ; q) \equiv Z\left(K ; Q^{(k)}+b^{(k)}, M-\left(Q^{(k)}+\right.\right.$ $\left.\left.b^{(k)}\right) \mid z^{\prime} ; q\right)$ and $Q_{N}^{(k)}(z ; q) \equiv Z\left(K^{-1} ; Q^{\prime(k)}+a^{(k)}, N-\left(Q^{\prime(k)}+a^{(k)}\right) \mid z ; q\right)$, entering (5.3), all arise as a solution to the same (that is, $k$-independent) set of recursion relations $(i=1, \ldots, n)[5]$

$$
\begin{aligned}
& P_{M}\left(z^{\prime} ; q\right)=P_{M-e_{i}}\left(z^{\prime} ; q\right)+z_{i}^{\prime} q^{-\frac{1}{z} K_{i i}+M_{i}} P_{M-K \cdot e_{i}}\left(z^{\prime} ; q\right), \\
& Q_{N}(z ; q)=Q_{N-e_{i}}(z ; q)+z_{i} q^{-\frac{1}{2} K_{i i}^{-1}+N_{i}} Q_{N-K-e_{e i}}(z ; q),
\end{aligned}
$$

where $e_{i}$ denotes the unit vector in the $i$-direction and where we have used

$$
\left[\begin{array}{l}
M \\
m
\end{array}\right]=\left[\begin{array}{c}
M-1 \\
m
\end{array}\right]+q^{M-m}\left[\begin{array}{c}
M-1 \\
m-1
\end{array}\right]
$$

For the application of Theorem 5.1 to affine Lie algebra characters let us consider the limiting form of (5.1) as $\boldsymbol{u} \rightarrow \infty$, that is,

$$
Z_{\infty}(K ; Q \mid z, q)=\lim _{u \rightarrow \infty} Z(K ; Q, u \mid z, q)=\sum_{m}\left(\prod_{i} z_{i}^{m_{i}}\right) \frac{q^{\frac{1}{2} m \cdot K \cdot m+Q \cdot m}}{\prod_{i}(q)_{m_{1}}}
$$

REMARK. The limiting UCPF's are not all independent. For instance, by using the simple relation $\left(1-q^{m}\right) /(q)_{m}=1 /(q)_{m-1}$ we find

$$
Z_{\infty}(K ; Q)=Z_{\infty}\left(K ; Q+e_{i}\right)+z_{i} q^{\frac{1}{2} e_{i} \cdot K \cdot e_{i}+Q \cdot e_{i}} Z_{\infty}\left(K ; Q+K \cdot e_{i}\right)
$$

By taking $\mathbf{M} \rightarrow \infty$ in (5.3) we obtain 
COROLLARY 5.2. Let $\left(\boldsymbol{Q}^{(k)}, \boldsymbol{a}^{(k)}, \boldsymbol{b}^{(k)}\right)$ be a Durfee system for $\boldsymbol{K} \in G L\left(n, \mathbb{Z}_{+}\right)$of length L. Define $Q^{\prime(k)}$ and $z_{i}^{\prime}$ by (5.2). We then have

$$
\begin{aligned}
& \sum_{k=0}^{L-1}\left(\prod_{i} z_{i}^{-Q_{i}^{(k)}}\right) q^{\frac{1}{2} Q^{(k)} \cdot K^{-1} \cdot Q^{(k)}+a^{(k)} \cdot b^{(k)}} Z_{\infty}\left(K ; Q^{(k)}+b^{(k)} \mid z^{\prime} ; q\right) \\
& \times Z_{\infty}\left(K^{-1} ; \boldsymbol{Q}^{\prime(k)}+\boldsymbol{a}^{(k)} \mid z ; q\right)=\frac{1}{(q)_{\infty}^{n}} \sum_{p \in \mathbb{Z}^{n}}\left(\prod_{i} z_{i}^{p_{i}}\right) q^{\frac{1}{2} p \cdot K^{-1} \cdot p} .
\end{aligned}
$$

Now suppose that the bilinear form $\boldsymbol{p} \cdot \boldsymbol{K}^{-1} \cdot \boldsymbol{p}$ is chosen in such a way that it equals the standard bilinear form on the weight lattice $\Lambda_{w}$ of a simple Lie algebra $g$ of rank $n$ and that the sum over $p \in \mathbb{Z}^{n}$ corresponds to the sum over the weight lattice. Then, provided $g$ is simply-laced, the right-hand side of (5.7) can be recognized as the Frenkel-Kac character of the sum of the level- 1 integrable highest weight modules of the affine Lie algebra $\widehat{\mathfrak{g}}$ (see, for example, [9]). (The irreducible characters can be recovered by suitably restricting the sum over $p$.) Thus, in such cases, Corollary 5.2 provides an expression for the level-1 characters of $\widehat{\mathfrak{g}}$ in terms of UCPF's based on the bilinear form constructed out of $K \oplus K^{-1}$. This has important applications in the study of quasiparticles in the conformal field theory descriptions of certain nonAbelian fractional quantum Hall states $[4,5]$. In fact, these applications were the main motivation for the present study.

As an example, consider $\mathfrak{g}=\mathfrak{s l}_{n+1}$. The weights $\left\{\epsilon_{1}, \ldots, \epsilon_{n+1}\right\}$, of the fundamental $(n+1)$-dimensional representation $L\left(\Lambda_{1}\right)$ of $\mathfrak{s l}_{n+1}$ satisfy $\epsilon_{i} \cdot \epsilon_{j}=\delta_{i j}-1 /(n+1)$. A suitable basis of the weight lattice $\Lambda_{\mathrm{w}}$ is given by the $\epsilon_{i}, i=1, \ldots, n$ (see Figure 5.1 for $\left.\mathfrak{s l}_{3}\right)$. Now note that $\left(\sum_{i} p_{i} \epsilon_{i}\right) \cdot\left(\sum_{j} p_{j} \epsilon_{j}\right)=p \cdot K^{-1} \cdot p$, where $K^{-1}$ is given by

$$
K^{-1}=\frac{1}{n+1}\left(\begin{array}{cccc}
n & -1 & \cdots & -1 \\
-1 & n & \cdots & -1 \\
\vdots & \vdots & \ddots & \vdots \\
-1 & -1 & \cdots & n
\end{array}\right)
$$

which has an inverse $K$ given by (3.6). The 'dual sector', defined by $K$, corresponds to a particular basis of the root lattice of $\mathfrak{s l}_{n+1}$ (see Figure 5.1 for $\mathfrak{s l}_{3}$ ). The weights of this basis are determined by (5.2).

Thus, the sum over $\boldsymbol{p} \in \mathbb{Z}^{n}$ is precisely over the weight lattice of $\mathfrak{s l}_{n+1}$ and combining Theorem 3.3 and Corollary 5.2 gives us an expression for the character of the (sum over all) level-1 integrable highest weight modules of $\widehat{\mathfrak{s l}}_{n+1}$. As a consistency check, note that

$$
\frac{1}{2} Q^{(k)} \cdot K^{-1} \cdot Q^{(k)}=\frac{k(n+1-k)}{2(n+1)}, \quad k=0, \ldots, n
$$




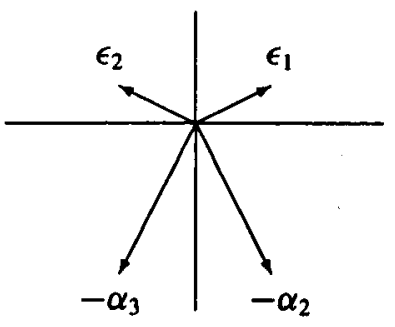

FIGURE 5.1. $\mathfrak{s l} l_{3}$ weights and roots

is indeed precisely the conformal dimension of the level-1 integrable highest weight module $L\left(\Lambda_{k}\right)$ of $\widehat{\mathfrak{s l}}_{n+1}$.

\section{Discussion and conclusions}

In this paper we have introduced higher dimensional analogues of the classical Durfee square formula (1.1) in the form of 'Durfee systems', we explained their correspondence to multipartitions, and gave a few examples. We have also remarked on the application of Durfee systems, in particular with regards to writing (chiral) characters of two-dimensional conformal field theories in UCPF form.

A number of obvious questions come to mind. Firstly, for which symmetric $K \in G L\left(n, \mathbb{Z}_{+}\right)$is it possible to find a Durfee system? It seems that this class of matrices is quite big. In fact, examples suggest that, provided det $K \geq 0$, a Durfee system always exists (see (2.3) for an example with det $K=0$ ). Secondly, how unique are Durfee systems for a given matrix $K$ ? Clearly they are not unique. For instance, in the case of $K=s / r$ (see Theorem 2.2) we can construct Durfee systems of length $L=m^{2} r s$ for all $m \in \mathbb{N}$ by taking $(r, s) \rightarrow(m r, m s)$ in (1.6) and (1.7). Similar constructions exist for the higher dimensional cases. Another source of non-uniqueness originates from possible symmetries of the matrix $K$. For example, interchanging the components of all vectors $\left(\boldsymbol{Q}^{(k)}, \boldsymbol{a}^{(k)}, \boldsymbol{b}^{(k)}\right)$ in Theorem 3.2, provides another Durfee system due to the $\mathbb{Z}_{2}$ permutation symmetry of the matrix $K$ in (3.4). Thirdly, for a given $K$, what is the minimal length $L_{\min }$ of a Durfee system? It seems that a special role is played by matrices for which $L_{\min }=\operatorname{det} K$, which seem to be closely related to matrices for which it is possible to choose a Durfee system for which $\boldsymbol{b}^{(k)}=0$ for all $k$. A large class of such matrices is provided by the shift deformations $K_{M, t}$ of the identity (see (4.1)) and, at least in two dimensions, it appears that such deformations exhaust all matrices $\boldsymbol{K}$ for which $L_{\min }=\operatorname{det} \boldsymbol{K}$. Finally, is it possible to give a more 'geometric' construction of the vectors $\left(Q^{(k)}, a^{(k)}, b^{(k)}\right)$ ? Again, in the case of matrices $K$ for which $L_{\min }=\operatorname{det} K$ it seems that the set of $Q^{(k)}$ is given by a set of coset representatives (with minimal non-negative components) of $\mathbb{Z}^{n}$ modulo 
the equivalences $\boldsymbol{m} \sim \boldsymbol{m}+\boldsymbol{K} \cdot \boldsymbol{e}_{i}(i=1, \ldots, n)$. Note that in the case of (3.6) the equivalence preserves the $\mathbb{Z}_{n+1}$ charge $q=\sum i m_{i}(\bmod n+1)$ of $m($ ' $n$-ality') and that we find one coset representative for each $q \in \mathbb{Z}_{n+1}$.

\section{References}

[1] G. E. Andrews, 'Generalizations of the Durfee square', J. London Math. Soc. 3 (1971), 563-570.

[2] - The theory of partitions, Encyclopedia Math. Appl. 2 (Addison-Wesley, Reading, 1976).

[3] - Partitions: Yesterday and today (New Zealand Mathematical Society, Wellington, 1979).

[4] E. Ardonne, P. Bouwknegt, S. Guruswamy and $K$. Schoutens, ' $K$-matrices for non-Abelian quantum Hall states', Phys. Rev. B61 (2000), 10298-10302 [cond-mat/9908285].

[5] E. Ardonne, P. Bouwknegt and K. Schoutens, 'Non-Abelian quantum Hall states-exclusion statistics, $K$-matrices and duality -', J. Stat. Phys. 102 (2001), 421-469.

[6] A. Berkovich and B. McCoy, 'The universal chiral partition function for exclusion statistics', in: Statistical Physics on the Eve of the 21 st Century (eds. M. T. Batchelor and L. T. Wille) Series on Adv. in Stat. Mech. 14 (World Scientific, Singapore, 1999) pp. 240-256. [hep-th/9808013].

[7] R. van Elburg and K. Schoutens, 'Quasi-particles in fractional quantum Hall effect edge theories', Phys. Rev. B58 (1998), 15704 [cond-mat/9801272].

[8] G. H. Hardy and E. M. Wright, An introduction to the theory of numbers (Oxford University Press, Oxford, 1960).

[9] V. G. Kac, Infinite dimensional Lie algebras (Cambridge University Press, Cambridge, 1985).

[10] J. J. Sylvester, A constructive theory of partitions, arranged in three acts, an interact and an exodion, Collected works, Vol. 4 (Cambridge University Press, Cambridge, 1912).

Department of Physics and Mathematical Physics

University of Adelaide

Adelaide SA 5005

Australia

e-mail: pbouwkne@physics.adelaide.edu.au 